\title{
An Integrated Algorithm of CCPP Task for Autonomous Mobile Robot under Special Missions
}

\author{
Caihong Li ${ }^{1}$, Zhiqiang Wang ${ }^{1}$, Chun Fang ${ }^{1}$, Zhenying Liang ${ }^{1}$, Yong Song ${ }^{2}$, Yibin Li ${ }^{2}$ \\ ${ }^{1}$ School of Computer Science and Technology, Shandong University of Technology, \\ Zibo, 255000, PR China \\ E-mail: lich@sdut.edu.cn \\ ${ }^{2}$ School of Control Science and Engineering, Shandong University, \\ Jinan, 250000, PR China \\ E-mail: songyong@sdu.edu.cn
}

Received 24 March 2018

Accepted 29 July 2018

\begin{abstract}
Due to the difficult problem of avoiding obstacles to achieve the complete coverage path planning (CCPP) for special missions, this paper introduces a novel integrated algorithm of CCPP for autonomous mobile robot under an obstacles-included environment. The algorithm combines cellular decomposition approach and the Standard map together for designing. The cellular decomposition approach is used to simplify the given workplace into smaller sub-regions for coverage via a chaotic path planner. The planner is constructed based on the chaotic Standard map at full mapping and produces the needed trajectories inside each decomposed sub-region. The simulation results verify the effectiveness of the designed method.
\end{abstract}

Keywords: Autonomous mobile robot; Complete coverage path planning (CCPP); Integrated algorithm; Chaotic path planner; Cellular decomposition approach; The Standard map

\section{Introduction}

With wide applications of the intelligent robot in domains, for example, the vacuum cleaning ${ }^{1}$, harvesting ${ }^{2}$, lawn mowing ${ }^{3}$, underwater sources exploring $^{4}$, unmanned aerial vehicles (UAV) ${ }^{5,6,7}$ and so on, one of the core technologies, complete coverage path planning (CCPP), has received extensive attentions CCPP is the task of determining a path that passes over all points of an area or volume of interest while avoiding obstacles ${ }^{8}$. In the above circumstances, only the complete coverage of the entire terrain is needed. While for some special missions, especially in the circumstances for military applications, such as the surveillance of terrains ${ }^{9}$, the terrain exploration for searching $^{10}$, demining ${ }^{11}$, and patrolling ${ }^{12}$, high unpredictability or randomness for the robot trajectories, as well as fast scanning of the workplace area are strongly required.

In 2001, Nakamura and Sekiguchi proposed a strategy to solve the above mentioned problems based on chaotic systems ${ }^{13}$. In that work, the chaotic behavior of the Arnold dynamical system was imparted to the mobile robot's motion control. Since then a great number of relative research works in the field of autonomous mobile robots have been presented, because the chaotic motion guarantees the scanning of the whole workspace without a terrain map or motion plan and can obtain unpredictable trajectories. The main characteristics of the chaotic systems are the topological transitivity and the sensitive dependence on initial conditions ${ }^{14}$. Due to topological transitivity, the chaotic mobile robot is guaranteed to patrol the whole surveillance region completely without repetitions. The sensitive dependence on initial conditions is a desirable 
characteristic for patrol robots, since the trajectory produced by the chaotic mobile robot is unpredictable or random ${ }^{15,16,17}$. Chaotic system is very different from the random signal. The chaotic motion is based on determinism, which in the case of mobile robots is an advantage. This happens because the behavior of a robot can be predicted in advance by the system designer.

The purpose of using chaotic systems in autonomous robots is realized by designing the chaotic path planner, which ensure chaotic motion. The trajectories produced by it are used to guide autonomous robots for exploration of a terrain for vigilance. In the literature, Lorenz dynamical system ${ }^{18,19}$, Standard or TaylorChirikov map ${ }^{9,10,21}$, Chua circuit ${ }^{22}$, Logistic map ${ }^{23}$, and double-scroll systems ${ }^{15,24,25}$ are some of the chaotic systems which have been commonly used for this purpose. We also constructed two chaotic motion path planners ${ }^{26,27}$, which were improved by arcsine or arccosine transformations, based on the Logistic map and Chebychev map, respectively, for an autonomous mobile robot to cover an unknown terrain completely, unpredictably, and evenly.

In the workplace without obstacles, the trajectories produced by the chaotic path planner can demonstrate the same performance as the original chaotic system, and meet the requirements of special missions. But it is difficult to design an obstacles avoiding strategy in an obstacles-included environment using the chaotic path planner while maintaining the chaotic characteristics of the coverage trajectories unchanged. Most of the researches now use the mirror mapping method to avoid obstacles for the chaotic mobile robot ${ }^{13,28,29,30}$. It really works when the distances between two adjacent iterative points produced by the chaotic system are very short, because it only requires the measurement of the local normal of the boundaries of workspace or obstacles when the robot comes close to them. But to the systems that the distances are very large, the method is not applicable because of the frequently used of the mirror mapping almost at each iterative step. So the trajectories produced by mirror mapping method will occupy larger part of the total coverage trajectories which can influence the chaotic characteristics of the coverage trajectories. Furthermore, it has to judge whether the produced trajectory will collide with the obstacles or not in each planning step, which can increase computation complex of the method and decrease the planning efficiency.
This paper introduces an integrated algorithm to solve the above problem based on the chaotic Standard map and cellular decomposition approach. The Standard map, or called Taylor-Chirikov, is a well known twodimensional mapping ${ }^{31}$. Martins-Filho and $\mathrm{Macau}^{9}$ proposed an ingenious path-planning mechanism where the sequence of intermediary goal positions were obtained using the map. We also proposed a fusion iterations strategy based on the map to generate a chaotic path planner of autonomous mobile robot for surveillance missions ${ }^{32,33}$. The Standard map is easy to be controlled for having a single parameter $K$ which determines its state. Its two variables $\left(x_{n}, y_{n}\right)$ can be directly mapped to the robot's position or coordinates in the workplace. Furthermore it has the better coverage property in running region for its full mapping characteristics at the chaotic state when $K \geq 8$. So we use the chaotic Standard map at $K=8$ to construct the chaotic path planner, and combine the cellular decomposition method to achieve the CCPP task under special missions.

The cellular decomposition method simplifies the complex coverage task into some simple sub-regions or cells for covering. Choset conjectured that most complete algorithms used an exact cellular decomposition of the free space, either explicitly or implicitly, to achieve coverage ${ }^{34}$. It partitions the given environment into several smaller sub-regions, and each sub-region is covered by the robot in a prescribed manner. Once each sub-region has been covered, the overall coverage task is realized.

In this research, the given workplace including obstacles has been divided into some cells, then the rectangular feasible sub-regions are formed by them based on a merging strategy. The chaotic path planner constructed by the Standard map realizes the coverage task in decomposed sub-regions. The size of each subregion should be in a rectangular shape to correspond to the running domain of the Standard map. The attractor characteristics of the chaotic path planner eliminates the detection of obstacles and boundaries of the workplace when the robot is covering inside a sub-region. After achieving the coverage task in one sub-region, the robot goes to another one by a safety zone, which is established to design the transition procedure between two adjacent sub-regions to avoid obstacles based on the attractor characteristics also. Avoidance of obstacles only occur during transition procedure and needs two 
steps at most to achieve one transition. Compared with mirror mapping method, the designed obstacles' avoidance method is very simple and efficient. The transition trajectories occupy little part of the whole coverage trajectories and have little effect to the chaotic characteristics of them. So under an obstacles-included environment, the constructed chaotic path planner can produce the coverage trajectories to achieve the CCPP task for special missions, with completeness, unpredictability, evenness and safety.

Some work above has been discussed in [33]. This paper further put forward the following new ideas:

(i) A systematic approach of merging strategy has been formed for constructing the rectangular iterative sub-regions. It simplifies the merging procedure and can be easily achieved by computer;

(ii) The attracting property of the chaotic Standard map is used for designing the transition procedure to facilitate the coverage procedure and improve the coverage efficiency.

(iii) The safe-zone is established to avoid the obstacle based on the attracting property of the chaotic Standard map. Only after two steps at most, the transition procedure can be achieved safely and conveniently.

The rest of the paper is organized as follows. The basic characteristics of the Standard map in the chaotic state are presented in Section 2. Section 3 constructs the chaotic path planner based on the Standard map at the fulling mapping state. The detailed designing procedure of the integrated algorithm based on cellular decomposition approach and the chaotic Standard map is described in Section 4. Section 5 illustrates a case to verify the designed algorithm. The last section outlines the conclusions and future work.

\section{Characteristics of the Standard Map}

The Standard map, or the Taylor-Chirikov, is a twodimensional areas preserving dynamical system ${ }^{35,36}$. Area-preserving mappings give rise to incredibly rich dynamics and mathematics ${ }^{37,38}$. The researchers commonly use the following discrete formula of the Standard map to model the chaotic path planner for an autonomous mobile robot:

$$
\left\{\begin{array}{l}
x_{n}=x_{n-1}+K \sin y_{n-1}, \\
y_{n}=y_{n-1}+x_{n}, \\
x_{n}=\bmod \left(x_{n}, 2 \pi\right), \\
y_{n}=\bmod \left(y_{n}, 2 \pi\right) .
\end{array}\right.
$$

Here $x_{n}$ is a periodic configuration variable (angular position), and $y_{n}$ is the momentum variable (angular speed), both computed mod $(2 \pi)$. The map has a single parameter, $K$, that represents the strength of the nonlinear kick. Randomly selected an initial point $\left(x_{0}, y_{0}\right)$, its next $n$ iterations, $\left(x_{n}, y_{n}\right)$, can be computed out step by step, where $n$ is the iterative time. Because the variables $\left(x_{n}, y_{n}\right)$ are both computed $\bmod (2 \pi)$, the range of them are bounded in $\left[\begin{array}{lll}0 & 2 \pi\end{array}\right]$.

\subsection{Bifurcation diagrams}

Parameter $K$ controls the dynamic properties of the Standard map. As it increases, the map evolves from double-periodic bifurcation to the chaotic state. Fig. 1 illustrates the bifurcation diagrams of the two variables with successive changing of the parameter $K$, respectively. When $K$ is greater than a certain value, the iterative values of $x_{n}$ and $y_{n}$ are full of the entire running domain, namely the system owns the completeness.

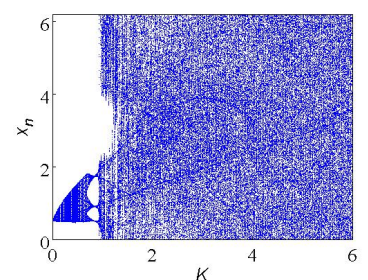

(a)

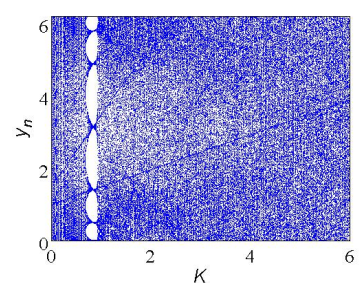

(b)
Fig. 1. Bifurcation diagram (a) $x_{n}$ vs. $K$ and (b) $y_{n}$ vs. $K$.

\subsection{Phase space}

Bifurcation diagrams of Fig. 1 can not distinguish the boundaries clearly among different states of the system due to resolution of the picture, so the method of phase space is used to further analyze the state changing. Fig. 2 shows the phase space of the map for parameter $K$ with varying values. As $K$ increases, the trajectories fill a larger region of the phase space. As $K=5$, seen in Fig. 2(c), only a small two blank windows are left. In Fig. 2(d), for $K=8$, one has an impression that chaos is complete, and the system is at a full mapping state in phase space. So parameter $K$ can assume an integer value equal or greater than 8 for the system to obtain a 
complete covering trajectories in its iterative region. It has a good distribution feature and is suitable for achieving the coverage mission.

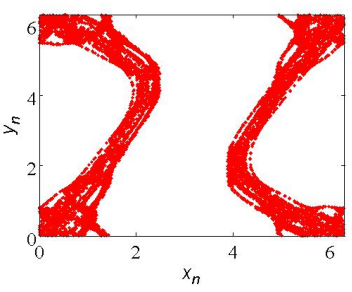

(a)

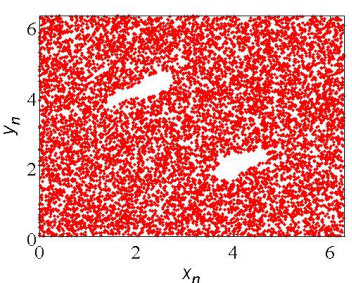

(c)

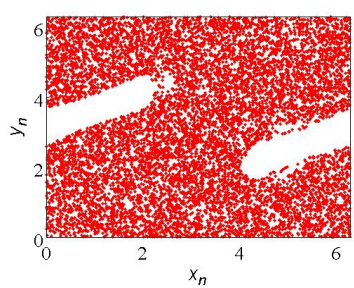

(b)

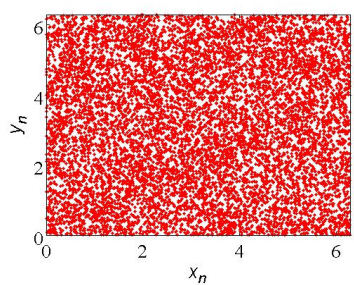

(d)
Fig. 2. Phase space of the the Standard map vs. $K$ (for $x_{0}=0.5$, $\left.y_{0}=0.5, \quad n=10,000\right) \quad$ (a) $K=1, \quad$ (b) $K=4, \quad$ (c) $K=5$ and (d) $K=8$.

\subsection{The largest Lyapunov exponent}

The largest Lyapunov exponent (LLE) is an important evaluation index of the chaotic state for a system. If LLE $>0$, the system can be assured to be chaotic ${ }^{39}$. And the greater the exponent is, the better the chaotic performance the system shows. Using 5,000 sampled time sequences of $x_{n}$ computed by formula (1), the LLE spectrum, shown in figure 3 , is calculated based on the reconstructed attractor method ${ }^{40}$.

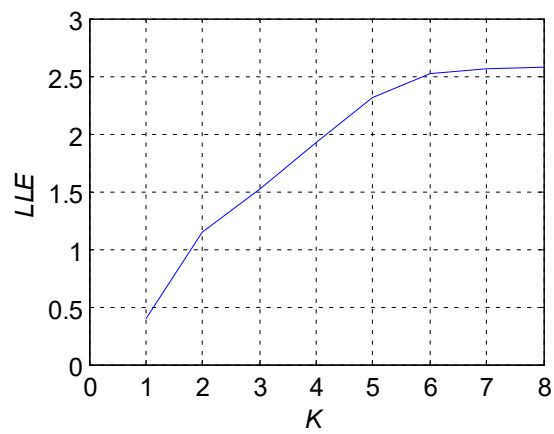

Fig. 3. Spectrum of LLE with parameter $K$

The picture shows that LLE is always greater than 0 when $K$ takes the positive integer. So the system keeps at the chaotic state owning these values, and has the maximum LLE as $K=8$, which is about 2.6. The system with this value has the best chaotic characteristics and is at the full mapping state, which is shown in Fig.2 (d). It is beneficial for the robot to produce the random and coverage trajectories. So the following map is used to achieve the CCPP task for the robot:

$$
\left\{\begin{array}{l}
x_{n}=x_{n-1}+8 \sin y_{n-1}, \\
y_{n}=y_{n-1}+x_{n}, \\
x_{n}=\bmod \left(x_{n}, 2 \pi\right), \\
y_{n}=\bmod \left(y_{n}, 2 \pi\right) .
\end{array}\right.
$$

\subsection{Distribution characteristics of the coverage trajectories}

The variables $\left(x_{n}, y_{n}\right)$ produced by Eq. (2) can be used as the planned intermediary waypoint of CCPP for autonomous mobile robot. Connection of all the subgoals according to the produced-order sequence constitute the coverage trajectories for robot to track. The planned results are shown in Fig. 4 and Fig. 5.

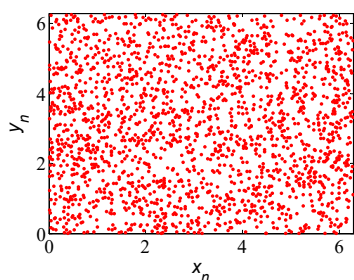

(a)

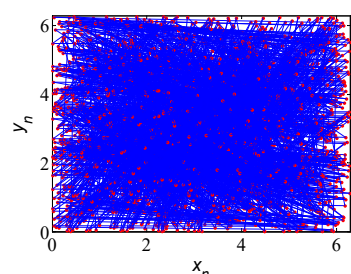

(b)
Fig. 4. Planned results of the coverage path planning at $n=2,000$ (a) planned sub-goals and (b) planned coverage trajectories.

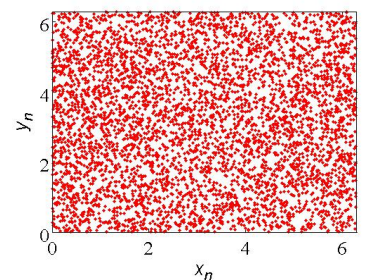

(a)

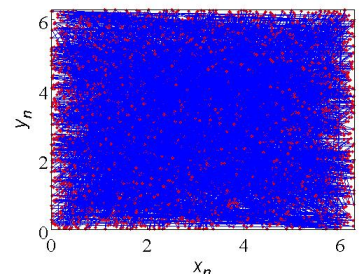

(b)
Fig. 5. Planned results of the coverage path planning at $n=5,000$ (a) planned sub-goals and (b) planned coverage trajectories.

From the above analysis, one can conclude that the planned sub-goals $\left(x_{n}, y_{n}\right)$ and coverage trajectories produced by the Standard map at $K=8$, have a very good uniformly distribution and can guarantee the 
complete coverage. It can meet the requirements, e.g., completeness, evenness, and unpredictability for special missions of CCPP task.

\section{The Chaotic Path Planner}

To cover a blank region of rectangular size, the trajectories produced by Eq. (2) can be used as the coverage path of CCPP task for autonomous mobile robot. Fig. 4 and Fig. 5 show that the operating range of $\left(x_{n}, y_{n}\right)$ is restricted in a rectangular scope $\left[\begin{array}{llll}0 & 2 \pi & 0 & 2 \pi\end{array}\right]$. By affine transformation based on Eq. (2), the running domain of $\left(x_{n}, y_{n}\right)$ can be mapped to a rectangular region of any size from the original one:

$$
\left\{\begin{array}{l}
x_{n}=x_{n-1}+8 \sin y_{n-1}, \\
y_{n}=y_{n-1}+x_{n}, \\
x_{n}=\bmod \left(x_{n}, 2 \pi\right), \\
y_{n}=\bmod \left(y_{n}, 2 \pi\right), \\
x_{n}=a+c \cdot x_{n}, \\
y_{n}=b+d \cdot x_{n} .
\end{array}\right.
$$

Where the coefficients $(a, b)$ are translation transformation parameters, and $(c, d)$ are scaling transformation parameters. Given any initial point $\left(x_{0}, y_{0}\right)$ in the region, time sequences, or the waypoint $\left(x_{n}, y_{n}\right)$ of each moment, can be calculated out step by step based on the above formula. Eq. (3) is the constructed chaotic path planner for autonomous mobile robot to achieve the CCPP task.

The designed chaotic path planner has attractor characteristics. Whether the initial point $\left(x_{0}, y_{0}\right)$ is within the iterative region or not, the successive iterative values $\left(x_{l} \sim x_{n}\right)$ and $\left(y_{l} \sim y_{n}\right)$ are all bounded between $(0,2 \pi)$ because of the modular calculation by $2 \pi$ in the original Standard map. So its iterative region also can be called as attracting area. After affine transformation, the attracting area can be mapped to a rectangle with any shape.

Suppose there is a workplace with size $10 \times 10$. Then the rectangular area $\left[\begin{array}{llll}0 & 10 & 0 & 10\end{array}\right]$ is its attracting region. The affine parameters $\left[\begin{array}{llll}a & b & c & d\end{array}\right]$ of the constructed

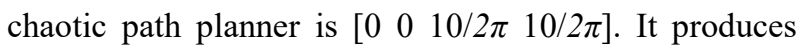
the coverage trajectories based on the given $\left(x_{0}, y_{0}\right)$ and Eq. (3). Fig. 6 illustrates the attractor characteristics of the chaotic path planner based on the planned coverage trajectories, where the circles "o" express the initial point $\left(x_{0}, y_{0}\right)$, the small little points mark other iterative values, and blue lines show the planned coverage trajectories.

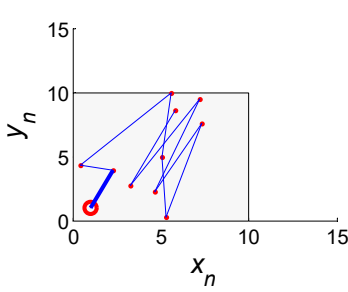

(a)

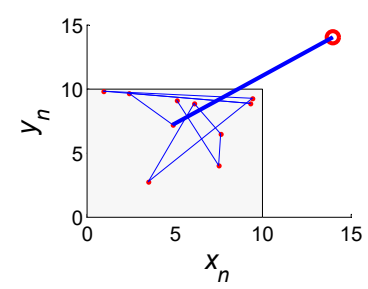

(c)

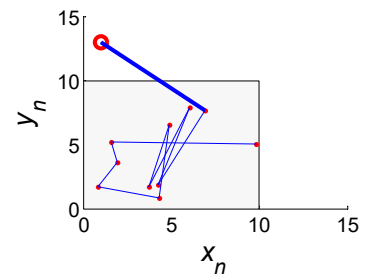

(b)

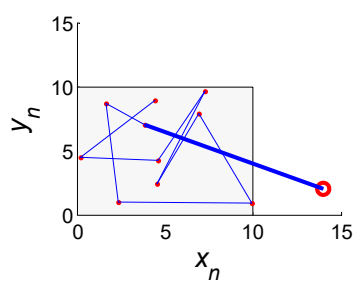

(d)
Fig. 6. Attractor characteristics at $n=10$ (a) $\left(x_{0}, y_{0}\right)=(1,1)$, (b) $\left(x_{0}, y_{0}\right)=(1,13)$, (c) $\left(x_{0}, y_{0}\right)=(14,14)$ and (d) $\left(x_{0}, y_{0}\right)=(14,2)$

In Fig. 6.(a), $\left(x_{0}, y_{0}\right)$ locates inside the workplace. No matter how many times the system iterates, the iterative values $\left(x_{n}, y_{n}\right)$ always lie in the workplace, and the robot can't collide with the boundaries of the workplace. So we needn't have to design the boundaries' avoidance method for the robot when it runs inside its attracting region. In Fig. 6.(b) (d), though $\left(x_{0}, y_{0}\right)$ locates outside the workplace, only after one iterative step, the robot goes into the attracting area $\left[\begin{array}{llll}0 & 10 & 0 & 10\end{array}\right]$. Using the property, we can design the transiting strategy to achieve the transition between two adjacent sub-areas.

\section{Integrated Algorithm Designing}

In a rectangular workplace including obstacles, cellular decomposition method is used to partition the workplace and some feasible iterative sub-regions are formed. When the autonomous mobile robot completes the coverage task by the chaotic path planner in each iterative sub-region in turn, the whole CCPP task realizes. The integrated algorithm includes partition of workplace, construction of the iterative sub-regions, establishment of the safety zones and the transition procedure design, formation of the coverage connectedpath, and the coverage procedure to achieve the CCPP task. All the partitioned and constructed sub-regions 
must be in a rectangular size to correspond to the shape of running domain or attracting region of the chaotic path planner.

\subsection{Partition of the workplace}

In an enclosed rectangular workplace including obstacles, the connected free areas without obstacles are partitioned into some smaller grids, labeled as $S_{x}$, along the boundaries of obstacles and workplace, and the subscript $x$ expresses the symbol of each $S_{x}$. Some parallel vertical and horizontal straight lines are used to segment the workplace. They extend to both sides along the obstacles' boundaries until meet the borders of workplace or the boundaries of other obstacles. Then the small adjacent rectangles $S_{x}$ are obtained from the crossing areas which are produced by the segment lines in feasible areas. The number of $S_{x}$ is related to the obstacles' number and positions in workplace.

\subsection{Construction of the iterative sub-regions}

In order to reduce the transition times, the number of the needed coverage sub-regions is the less, the better. Here we define a iterative sub-region, labeled as $S_{I_{-} x}$, to merge the partitioned grids $S_{x}$. There are many methods that can be used. In our previous work ${ }^{33}$, the corner rectangles were defined to design the method. Here, another simpler merge algorithm is designed. First select a merging direction and a group of parallel lines based on it. The merging direction can be chosen from the followed ones, namely from top to bottom, bottom to top, left to right or right to left. The parallel lines include the above partition segment lines and the border lines of workplace. Then a rectangular iterative subregion $S_{I_{-} x}$ can be constructed by all $S_{x}$ between two adjacent parallel lines. The final number of $S_{I_{-} x}$ is same as other methods obtained and has the least value. The detailed strategy is described in Algorithm 1.

The functions contained in Algorithm 1 are introduced as follows:

- Direction_select (i): The function is to select a merging direction. There are four directions for being selected:

$i=1$, Direction_select $(1)=1$, from top to bottom; $i=2$, Direction_select $(2)=2$, from bottom to top; $i=3$, Direction_select (3) $=3$, from left to right; $i=4$, Direction_select $(4)=4$, from right to left.
- Line_define $(L(x), N)$ : The function is to define a group of segment lines based on the above segmentation strategy, where $N$ is the total number.

- Judge_adjacent $(L(n), L(m))$ : The function is to judge the two lines $L(n)$ and $L(m)$ are adjacent or not. If yes, the function is equal to 1 ; otherwise is 0 .

- Merge ( $\left.S_{x}, L(n), L(m)\right)$ : The function is to merge all the $S_{x}$ between the two adjacent lines $L(n)$ and $L(m)$ into a $S_{I_{-} x}$.

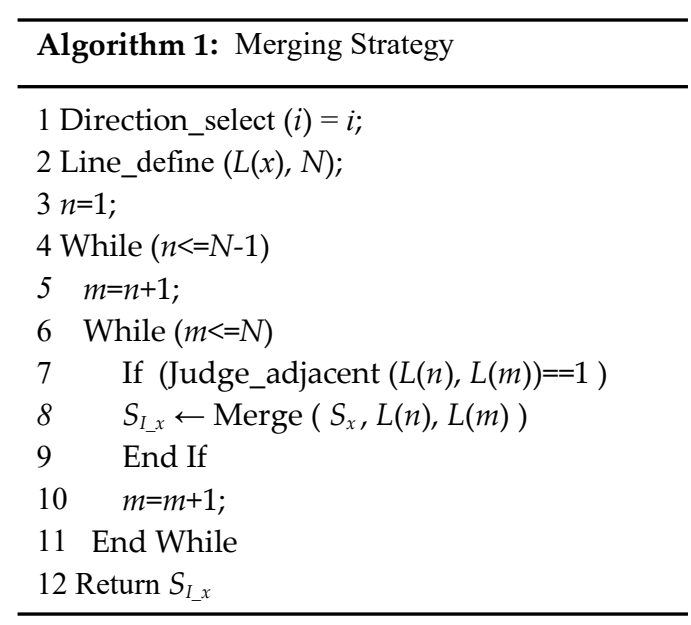

\subsection{Establishment of the safety zones and the transition procedure design}

When the robot finishes the CCPP task in one $S_{I_{-} x}$, it should transfer to the adjacent one to continue working. Based on the attractor characteristics discussed in Section 3, the robot can achieve mutual transitions between two adjacent $S_{I_{-} x}$ via the trajectories produced by the chaotic path planner directly and easily. But when there is an obstacle nearby, the direct transition may cause collision of the robot with the obstacle. So, the safety zone, labeled as $S_{f_{-}}$, is set up to solve the problem. $S_{f_{x}}$ is one of the partitioned grids $S_{x}$ between two adjacent $S_{I_{-}}$. It is defined as follows.

Definition 1 ( Safety zone). The grid $S_{x}$ which is located inside one $S_{I_{-} x}$ and has part overlap border with the adjacent $S_{I_{-} x}$ is called the safety zone $S_{f_{-}}$.

Suppose there are two adjacent iterative sub-regions which are labeled as $S_{I_{-} x}$ and $S_{I_{-} y}$, respectively. The transition procedure from $S_{I_{-} x}$ to $S_{I_{-} y}$ based on safety zone $S_{f_{-} x}$ and the attractor characteristics of the chaotic path planner is designed. The flowchart is shown in Fig. 7. It is only in the case that the final iterative point $\left(x_{t}, y_{t}\right)$ 
produced by the planner in $S_{I_{-} x}$ ends in $S_{f_{-} x}$, the robot can directly starts the iteration procedure of the chaotic path planner in $S_{I_{-} y}$, and the coverage trajectories transit from $S_{I_{-} x}$ to $S_{I_{y} y}$ directly and safely without the help of $S_{f_{-} x}$. While in other cases, in order to avoid the obstacle, the robot has to pass $S_{f_{-}}$to achieve the transition from $S_{I_{-} x}$ to $S_{I_{-} y}$.

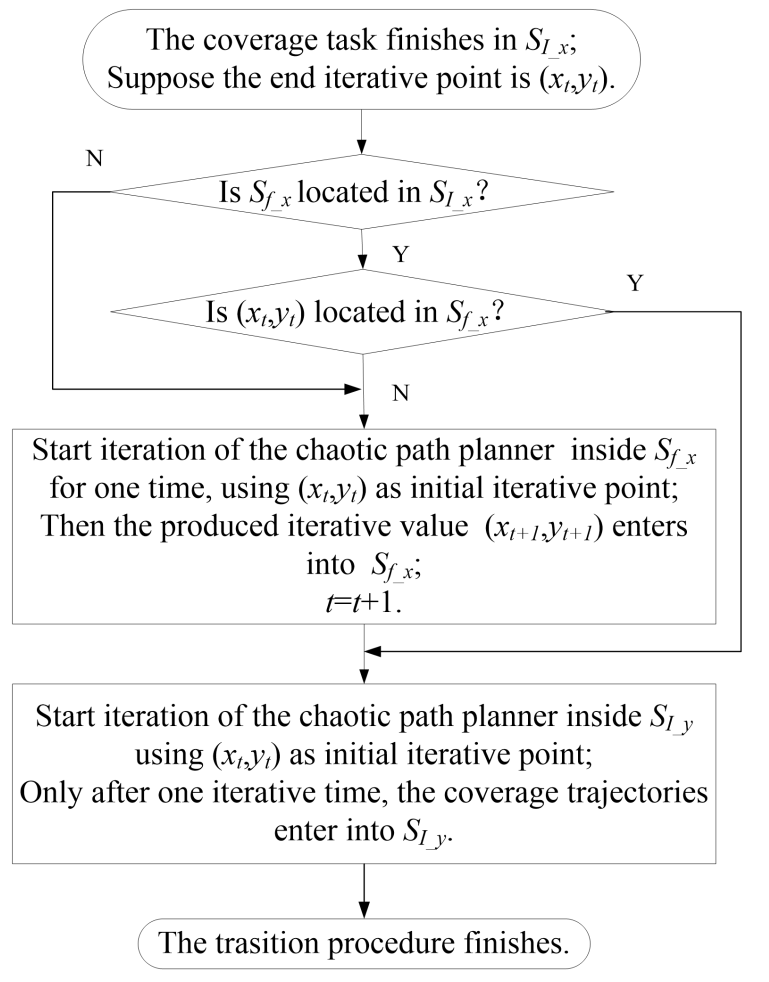

Fig. 7. Flowchart of the transition procedure

\subsection{Formation of the coverage connected-path}

The robot has to cover each $S_{I_{-} x}$ according to a connected-order. The order can be set in a constant sequence to guarantee the complete coverage and avoid overlapping coverage, or in a random sequence to acquire more unpredictable trajectories. A constant sequence should satisfy the following conditions:

- The path must include every $S_{I_{-} x}$ to guarantee the complete coverage.

- The number of $S_{I_{-} x}$ in the path should be as little as possible to avoid overlapping of coverage.

Though the question can be formulated as a TSP task, it has some difference. For the CCPP problem, each node (sub-region) in the route can be visited for several times to finish the coverage task instead one if only each sub-region owns relatively the same coverage evenness.

\subsection{Covering procedure}

According to the above designed algorithm, CCPP task in workplace can be achieved as follows.

(i) Initialization of CCPP task.

(a) Compute the affine parameters $[a b c d]$ of all $S_{I_{-} x}$ and $S_{f_{-} x}$, and construct the chaotic path planner of them based on Eq. (3);

(b) Set up the total loop time $N_{\text {Loop }}$. Each $S_{I_{-}}$being covered for one time is a loop;

(c) Set up the total iterative time $N_{I}$ in one $N_{\text {Loop }}$ according to the task requirements;

(d) Compute iterative time $n_{I_{-} x}$ of $S_{I_{-} x}$ related to $N_{l}$ based on their size proportion to the total free area, respectively;

(e) Select the covering direction $P . P=1$ : clockwise, $P=0$ : counterclockwise;

(f) Select an original point $\left(x_{0}, y_{0}\right)$ randomly, and determine the $S_{I_{-}}$in which the point is located;

(g) Set a parameter $N_{T}$ to record the transition time between two adjacent $S_{I_{-} x}$ during CCPP task, the initial value $N_{T}=0$.

(ii) Start the chaotic path planner based on $\left(x_{0}, y_{0}\right)$ and produce the covering trajectories in $S_{I_{-} x}$ until the sub-task of CCPP realizes inside.

(iv) Select the next $S_{I_{-} x}$ according to the designed coverage connected-path.

(v) Realize the transition procedure based on the designed method mentioned in Section 4.3.

(vi) Repeat the above procedure (ii) (iv) until the covering trajectories return to the start $S_{I_{-} x}$ and one loop ends.

(vii) Repeat the above procedure (ii) (v) until all loops end, and the whole CCPP task achieves.

\section{Verification of the Proposed Algorithm}

Here we assume a case to verify the proposed integrated method for achieving CCPP task. For the sake of simplicity, we select the most common used obstacles to illustrate the feasibility of the algorithm.

Case: as shown in Fig. 8.(a), the size of workplace is $10 \times 10$. There is a dark gray obstacle which size is $4 \times 4$ seated at the center of workplace. Its coordination of left-bottom corner is $(3,3)$. The requirement is to compute the complete coverage trajectories for CCPP task while avoiding the obstacle, and meet the demands for the special missions. 


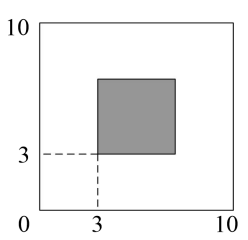

(a)

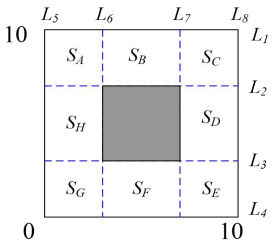

(b)

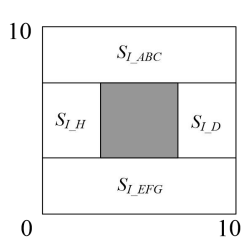

(c)
Fig. 8. Workplace and the decomposition result (a) original workplace, (b) decomposition into grids $S_{x}$, and (c) construction of the iterative sub-regions $S_{I_{x}}$

The research resolves the problem based on the strategy discussed in Section 4 step by step.

Fig. 8.(b) shows the decomposition result. The lines $L_{1} \sim L_{4}$ are a group of horizontal parallel segmentation lines, while $L_{5} \sim L_{8}$ are the vertical ones, among which $L_{1}$, $L_{4}, L_{5}$ and $L_{8}$ are the boundary lines of workplace. After segmentation, the feasible area is divided into eight small adjacent grids $S_{x}$, along the boundaries of obstacles and workplace, labeled as $S_{A}, S_{B}, S_{C}, S_{D}, S_{E}, S_{F}$, $S_{G}$ and $S_{H}$, respectively.

Select the top-bottom direction as merging direction, four iterative sub-regions, namely $S_{I_{-} A B C}, S_{I_{-} H}, S_{I_{-} D}$, and $S_{I_{-} E F G}$ are constructed respectively, which is shown in Fig. 8.(c), where $S_{I_{-} A B C}$ is formed by $S_{A}, S_{B}$, and $S_{C}$, and $S_{I_{-} E F G}$ is formed by $S_{E}, S_{F}$ and $S_{G}$.

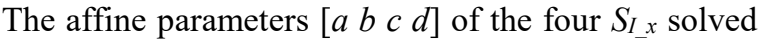
by Eq. (2) for the chaotic path planner are illustrated in Table 1. They are used to construct the chaotic path planner based on Eq. (3) to produce the coverage trajectories inside each $S_{I_{-} x}$, and part traversing trajectories for transition between two adjacent $S_{I_{-} x}$.

Table 1 Affine transformation parameters $S_{I_{-} x}$

\begin{tabular}{ccccc}
\hline \multirow{2}{*}{$S_{I_{-} x}$} & \multicolumn{4}{c}{ Affine transformation parameters } \\
\cline { 2 - 5 } & $a$ & $b$ & $c$ & $d$ \\
\hline$S_{I_{-} A B C}$ & 0 & 7 & $10 / 2 \pi$ & $3 / 2 \pi$ \\
$S_{I_{-} H}$ & 0 & 3 & $3 / 2 \pi$ & $4 / 2 \pi$ \\
$S_{I_{-} D}$ & 7 & 3 & $3 / 2 \pi$ & $4 / 2 \pi$ \\
$S_{I_{E F G}}$ & 0 & 0 & $10 / 2 \pi$ & $3 / 2 \pi$ \\
\hline
\end{tabular}

The safety zone $S_{f_{-} x}$ between two adjacent $S_{I_{-} x}$ is determined based on their connection relationship and Definition 1. All $S_{f_{-} x}$ solved according to Fig. 8(b) and Fig. 8(c) are illustrated in Table 2. The affine

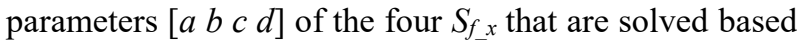
on Eq. (2) are listed in Table.3.
Table $2 S_{f_{-} x}$ between two adjacent $S_{I_{-} x}$

\begin{tabular}{cc}
\hline Adjacent $S_{I_{-} x}$ & $S_{f_{-} x}$ \\
\hline$S_{I_{-} A B C}$ and $S_{I_{-} D}$ & $S_{f_{-} C}$ \\
$S_{I_{-} D}$ and $S_{I_{-} E F G}$ & $S_{f_{-} E}$ \\
$S_{I_{-} E F G}$ and $S_{I_{-} H}$ & $S_{f_{-} G}$ \\
$S_{I_{-} H}$ and $S_{I_{-} A B C}$ & $S_{f_{-} A}$ \\
\hline
\end{tabular}

Table 3 Affine transformation parameters of $S_{f_{-} x}$

\begin{tabular}{ccccc}
\hline \multirow{2}{*}{$S_{f_{-} x}$} & \multicolumn{4}{c}{ Affine transformation parameters } \\
\cline { 2 - 5 } & $a$ & $b$ & $c$ & $d$ \\
\hline$S_{f_{-} C}$ & 7 & 7 & $3 / 2 \pi$ & $3 / 2 \pi$ \\
$S_{f_{-} E}$ & 7 & 0 & $3 / 2 \pi$ & $3 / 2 \pi$ \\
$S_{f_{-} G}$ & 0 & 0 & $3 / 2 \pi$ & $3 / 2 \pi$ \\
$S_{f_{A}}$ & 0 & 7 & $3 / 2 \pi$ & $3 / 2 \pi$ \\
\hline
\end{tabular}

They will be used to construct the chaotic path planner based on Eq. (3) to produce the transition trajectories between two adjacent $S_{I-x}$.

One of the transition procedures, from $S_{I_{-} A B C}$ to $S_{I_{-} D}$, is illustrated, where $S_{f_{C}}$ is their safety zone. The simulation result is shown in Fig. 9. The iterative time $n=30$, and the original point $\left(x_{0}, y_{0}\right)=(3,8)$, which is marked as purple "o" in the figure. The chaotic path planner produces the coverage trajectories in $S_{I_{-} A B C}$ based on $\left(x_{0}, y_{0}\right)$, which is shown in Fig. 9.(a). After achieving the coverage task, the final iterative point $\left(x_{t}\right.$, $\left.y_{t}\right)$ marked as red “*”, ending at (4.9972,7.3845), isn't located in $S_{f_{-} C}$. So $S_{f_{-} C}$ has to be used as the transition region to avoid possible collision of the obstacle. In Fig. 9.(b), start the iterative procedure of the chaotic path planner in $S_{f_{-} C}$ for one time based on $\left(x_{t}, y_{t}\right)$, the next iterative point $\left(x_{t+1}, y_{t+1}\right)$ is immediately attracted to $S_{f_{-} C}$, which is marked as a red " $\diamond$ ". Then based on $\left(x_{t+1}, y_{t+1}\right)$, start the iterative procedure in $S_{I_{-} D}$, and after only one time the coverage trajectory enters into it and the transition procedure ceases, which is shown in Fig. 9.(c). The red lines in the figure show the transition trajectories.

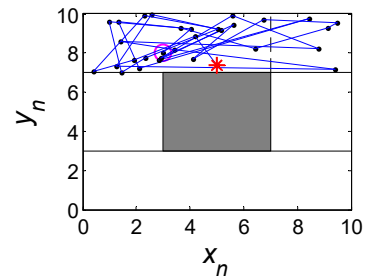

(a)

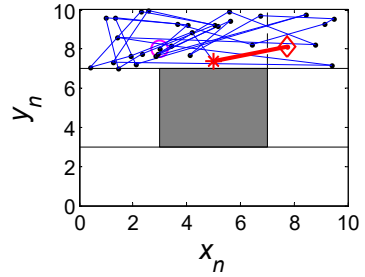

(b)
Fig.9. Transition procedure from $S_{I_{-} A B C}$ to $S_{I_{-} D}$ 


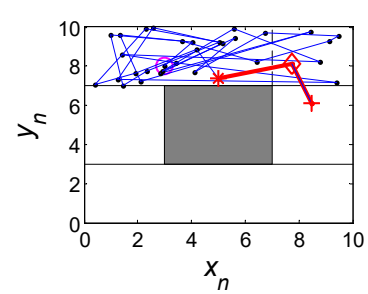

(c)

Fig.9. cont. Transition procedure from $S_{I_{-} A B C}$ to $S_{I_{-} D}$

Fig. 9 shows that the transition procedure achieves conveniently and safely. Only after two steps at most, the robot can travel from one $S_{I_{-} x}$ to its adjacent one. The linkage points that collecting the coverage trajectories are produced randomly by the chaotic path planner instead of designing deliberately. The coverage trajectories can avoid obstacle easily because of the installment of the safety zone $S_{f_{-} x}$, here is $S_{f_{-} C}$. Without it, the robot will collide with the obstacle possibly, which is shown as the red lines in Fig. 10. Of course,

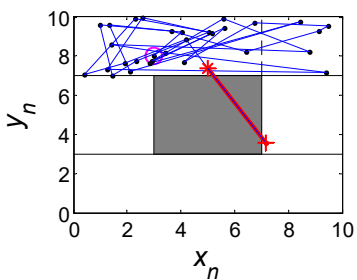

Fig. 10. Circumstance of obstacle collision without using $S_{f_{-} C}$

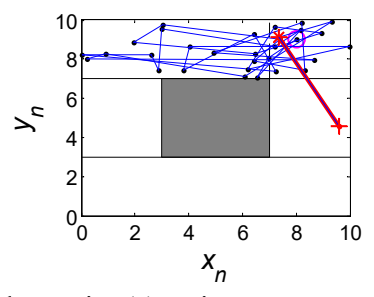

Fig. 11. Circumstance of $\left(x_{t}, y_{t}\right)$ ending in $S_{f_{-} C}$ when $\left(x_{t}, y_{t}\right)$ ends in $S_{f-C}$, we can start the iteration of $S_{I D}$ directly, and transition procedure achieves by only one step without colliding with the obstacle. Fig. 11 shows the case, where $\left(x_{0}, y_{0}\right)=(8,9)$. Safety zone is unused under this circumstance.

A coverage connected-path is formed to guide the coverage direction for robot. Fig. 12 shows a constant linkage one. It links the four $S_{I_{-} x}$ in a circle, where the solid arrows indicate clockwise direction, while the dotted arrows indicate counterclockwise direction.

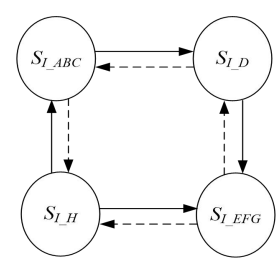

Fig. 12. The coverage connected-path
Based on the constructed chaotic path planner and the coverage connected-path, the covering procedure algorithm is designed, which is shown in Fig. 13.

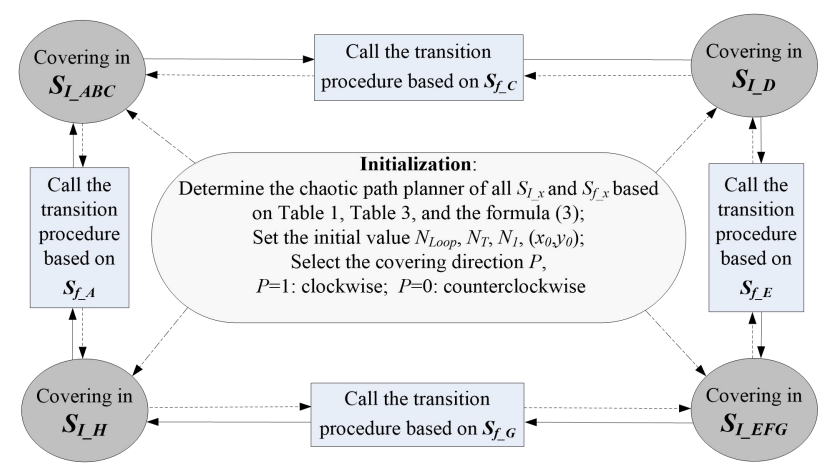

Fig. 13. The covering procedure

The algorithm has been simulated with different original point $\left(x_{0}, y_{0}\right)$, iterative time $N_{l}$, and loop time $N_{\text {Loop }}$. Select the coverage direction $P=1$. The original value $N_{T}=0$. According to the designed transition procedure, the possible value of $N_{T}$ after achievement of CCPP task in one loop will assume 4, 5, or 6. Different covering results can be obtained by changing the iterative times of $N_{l}$ and $N_{\text {Loop }}$.

Fig. 14 and Fig. 15 shows the coverage results in one loop, namely $N_{\text {Loop }}=1$. The marks and lines have the same meaning as the above figures. The results show that from any original point $\left(x_{0}, y_{0}\right)$, the robot can achieve the CCPP task conveniently, quickly, and safely by a limited transition time, based on the trajectories produced by the chaotic path planner. The larger the iterative time is, the better the coverage effect can get. Compared with Fig. 14, where $N_{l}=420$, results in Fig. 15 show a better evenness and completeness at $N_{l}=8400$. In one loop, whether the $N_{l}$ is, the $N_{T}$ remains nearly a constant value.

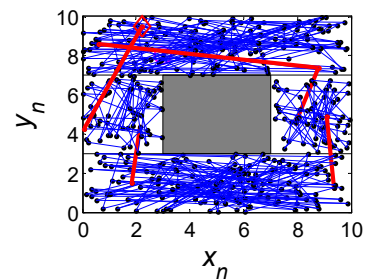

(a)

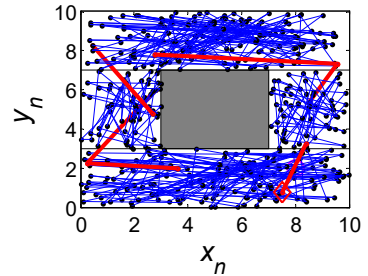

(b)
Fig. 14. The coverage trajectories at $N_{l}=420, N_{\text {Loop }}=1$ $\left(x_{0}, y_{0}\right)=(3,8), N_{T}=5$ and (b) $\left(x_{0}, y_{0}\right)=(8,2), N_{T}=6$. 


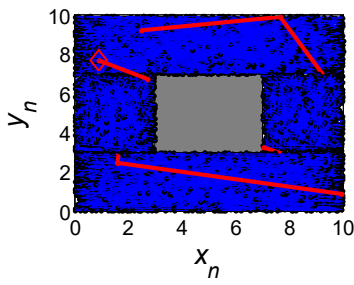

(a)

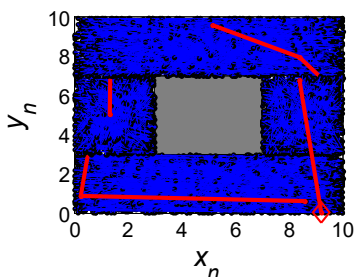

(b)
Fig. 15. The coverage trajectories at $N_{l}=8400, N_{\text {Loop }}=1$ (a) $\left(x_{0}, y_{0}\right)=(3,8), N_{T}=6$ and (b) $\left(x_{0}, y_{0}\right)=(8,2), N_{T}=6$.

Though the original point $\left(x_{0}, y_{0}\right)$ can be set randomly, the covering results remain the similar coverage characteristics at the same iterative times, especially when the iterative time assumes a larger value. Because there are little transition times during coverage procedure, the influence of them can be ignored. So the coverage trajectories show almost the same characteristics as the chaotic Standard map, and can satisfy the needs of special missions.

We can further improve the random characteristics of coverage trajectories by increasing the transition frequency among $S_{I_{-} x}$. Fig. 16 has the same total

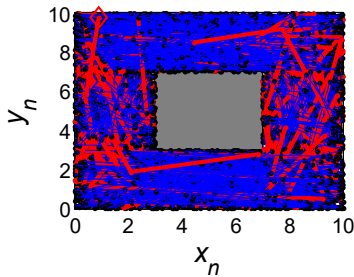

(a)

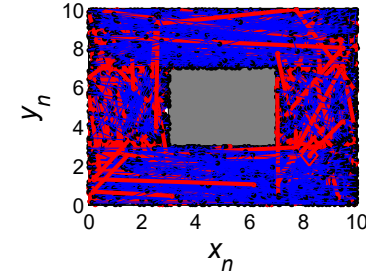

(b)
Fig. 16. The coverage trajectories at $N_{1}=84, N_{\text {Loop }}=100$ (a) $\left(x_{0}, y_{0}\right)=(3,8), N_{T}=529$ and (b) $\left(x_{0}, y_{0}\right)=(8,2), N_{T}=536$.

coverage times as Fig. 15, but in 100 loop times. The transition trajectories, namely the red lines, increase obviously. The circumstance may spoil some distribution characteristics of the coverage trajectories produced by the chaotic path planner. This phenomenon can be coordinated according to the task requirements.

Besides the complex computation and low planning efficiency, the common used mirror mapping method is unavailable to the chaotic path planner constructed by the Standard map because of the long distances between two adjacent iterative points produced by the planner $^{29}$, which can cause the frequently collision with the obstacles. Under this circumstance, the trails produced by the mirror mapping will occupy most part of the coverage trajectories which can totally destroy the chaotic characteristics of them.

\section{Conclusion}

Taking advantage of the chaotic characteristics of the Standard map, an integrated algorithm has been proposed based on cellular decomposition approach in the obstacles-included environment. The cellular decomposition simplifies the CCPP task into some subregions coverage task, using trajectories produced by the chaotic path planner based on the Standard map. The attractor characteristics of the Standard map can deduce the designing complex of obstacles avoidance strategy. The planned coverage trajectories demonstrate almost the same characteristics as the Standard map, such as the completeness, evenness, and randomness or unpredictability, to satisfy the needs of special missions. The designed method owns the following advantages:

- No designated linkage points are needed in transition procedure between two adjacent $S_{I_{-} x}$. They are produced by the chaotic path planner randomly in real time.

- Only after two steps at most, the robot can achieve the transition procedure between two adjacent $S_{I_{-}}$, so the transition trajectories have little influence to the chaotic characteristics of coverage trajectories.

- The obstacles' avoidance method is very simple. Due to the attractor characteristics of the Standard map, the robot can work in a $S_{I_{-} x}$ forever without going out or colliding with the boundaries of workplace. So no edges' detection of obstacles and the boundaries of workplace are needed when the robot runs inside a $S_{I_{-} x}$. Only during transition procedure, the problem of obstacle avoidance is considered.

- Establishment of the safety zone between two adjacent $S_{I_{-} x}$ makes transition procedure safe and simple based on the attractor characteristics of the Standard map.

There are also many work to be further researched in the future:

- The size of non-rectangular obstacles should be considered. So we have to study the mapping strategy of the Standard map to any size area.

- For a target workplace including complex obstacles, the decomposition and transition strategy for the sub-regions have to be explored in depth. 


\section{Acknowledgments}

This work is supported by the National Natural Science Foundation (NNSF) of China (61473179, 61602280, 61573213); The Natural Science Foundation of Shandong province (ZR2014FM007, ZR2014FQ028, ZR2015CM016 and 2016GGX101027).

\section{References}

1. E. Park, K. J. Kim, P. Del, et al, Energy efficient cmplete coverage path planning for vacuum cleaning robots, Lecture Notes in Electrical Engineering, Future Information Technology, Application, and Service. 164(1) (2012) 23-31.

2. T. Oksanen and A. Visala, Coverage path planning algorithms for agricultural field machines, Journal of Field Robotics. 26(8) (2009) 651-668.

3. J. Ousingsawat and M. G. Earl, Modified lawn-mower search pattern for areas comprised of weighted regions, Proceedings of the 2007 American Control Conference. (New York City, USA, 2007), 918-923.

4. E. Galceran and M. Carreras, Planning coverage paths on bathymetric maps for in-detail inspection of the ocean floor. International Conference on Robotics and Automation. (Karlsruhe, 2013), 4159-4164.

5. M. Torres, D. A. Pelta, J. L. Verdegay, et al, Coverage path planning with unmanned aerial vehicles for $3 \mathrm{D}$ terrain reconstruction, Expert Systems with Applications, 55(15) (2016), 441-451.

6. ZH. Zheng, Y. Liu, and X. Y. Zhang, The more obstacle information sharing, the more effective real-time path planning?. Knowledge-Based Systems, 114 (2016), 36-46.

7. Y. Liu, ZH. Zheng, and K.Y.Cai, Bi-level programming based real-time path planning for unmanned aerial vehicles. Knowledge-Based Systems, 44 (2013) 34-47.

8. E. Galceran and M. Carreras, A survey on coverage path planning for robotics, Robotics and Autonomous Systems. 61(12) (2013) 1258-1276.

9. L. S. Martins-Filho and E. E. N. Macau, Patrol mobile robots and chaotic trajectories, Mathematical Problems in Engineering. 2007 (2007) 1-13

10. P. Sooraksa and K. Klomkarn. No-CPU chaotic robots: from classroom to commerce, IEEE Circuits and Systems Magazine. 10(1) (2010) 46-53.

11. J. Prado and L.Marques, Energy efficient area coverage for an autonomous demining robot, Advances in Intelligent Systems and Computing. 253 (2014) 459-471.

12. K.S. Hwang, J.L. Lin, H.L. Huang, Dynamic patrol planning in a cooperative multi-robot system, 14th FIRA RoboWorld Congress on Next Wave in Robotics, Communications in Computer and Information Science. 212 (Kaohsiung, Taiwan, 2011) 116-123.

13. Y. Nakamura, A. Sekiguchi, The chaotic mobile robot, IEEE Transaction on Robotics and Automation. 17(6) (2001) 898-904
14. E.N. Lorenz. The essence of chaos. Meteorological Publishing House. (Beijing, China, 1997) 186-189, (in Chinese).

15. C. K. Volos, I. M. Kyprianidis, and I. N. Stouboulos. A chaotic path planning generator for autonomous mobile robots, Robotics and Autonomous Systems. 60(4) (2012) 651-656.

16. A. I. Zhu and H. Leung, Cooperation random mobile robots based on chaos synchronization, In Proceedings of the 4th IEEE International Conference on Mechatronics. (Kumamoto-shi, Japan, 2007) 1-5.

17. K. Fallahi and H. Leung, A cooperative mobile robot task assignment and coverage planning based on chaos synchronization, International Journal of Bifurcation and Chaos. 20(1) (2010) 161-176.

18. L. S. Martins-Filho and E. E. N. Macau, Patrol mobile robots and chaotic trajectories, Mathematical Problems in Engineering, 2007 (2007) 1-13.

19. D.I. Curiac and C. Volosencu, Developing 2D trajectories for monitoring an area with two points of interest, In: Proc. of the 10th WSEAS Int. Conference on Automation and Information. (Prague, Czech Republic, 2009) 366369.

20. L.S. Martins-Filho and E.E.N. Macau, Trajectory planning for surveillance missions of mobile robots, Studies in Computational Intelligence. (Berlin, Heidelberg, 2007) 109-117.

21. L.S. Martins-Filho, E.E.N. Macau, R. Rocha, et al, Kinematic control of mobile robots to produce chaotic trajectories, ABCM Symposium Series in Mechatronics. 2 (2006) 258-264.

22. Ch.K. Volos, Motion direction control of a robot based on chaotic synchronization phenomena, Journal of Automation. Mobile Robotics and Intelligent Systems. 7 (2) (2013) 64-69

23. C. Y. Qiu, Bare bones particle swarm optimization with adaptive chaotic jump for feature selection in classification, International Journal of Computational Intelligence Systems. 11 (2018) 1-14.

24. Ch.K. Volos, I.M. Kyprianidis and I.N. Stouboulos, Motion control of robots using a chaotic truly random bits generator, Journal of Engineering Science and Technology Review. 5 (2) (2012) 6-11.

25. Ch.K. Volos, N. Bardis, I.M. Kyprianidis, et al., Motion control of a mobile robot based on double-scroll chaotic circuits, WSEAS Transactions on Systems. 11 (9) (2012) 479-488.

26. C. H. Li, F. Y. Wang, L. Zhao, et al. An improved chaotic motion path planner for autonomous mobile robots based on logistic map, International Journal of Advanced Robotic Systems. 10 (2013) 1-9.

27. C. H. LI, Y. SONG, F. Y. WANG, et al. A chaotic coverage path planner of the mobile robot based on the chebychev map for special missions, Frontiers of Information Technology \& Electronic Engineering. 18(9) (2017) 1305-1319. 
28. Y. Bae, M. Lee, and T. M. Gatton, An obstacle avoidence method for chaotic robots using angular degree limitions, Journal of Security Engineering . 2(3) (2005) 244-250.

29. Y. Bae, Target searching method in the chaotic mobile robot, The 23rd Digital Avionics Systems Conference. (Salt Lake City, UT, USA, 2004) 12.D.7-1 -12.D.7-9.

30. A. A. Fahmy, Performance evaluation of chaotic mobile robot controllers, International Transaction Journal of Engineering, Management, \& Applied Sciences \& Technologies. 3(2) (2012) 145-158.

31. G. Contopoulos, Order and chaos in dynamical systems, Milan Journal of Mathematics. 77(1) (2009) 101-126.

32. C. H. Li, Y. Song, F. Y. Wang, et al. Chaotic path planner of autonomous mobile robots based on the Standard map for surveillance missions, Mathematical Problems in Engineering. 2015 (2015) 1-12.

33. C. H. Li, Zh. Q. Wang, F.Y. Wang, et al. A CCPP algorithm based on the Standard map for the mobile robot, 2017 Chinese Automation Congress, (Jinan, China, 2017), 4963-4968.

34. H. Choset, Coverage for robotics-a survey of recent results, Annals of Mathematics and Artificial Intelligence. 1 (2001) 113-126.

35. M. Shamis and T. Spencer, Bounds on the Lyapunov exponent via crude estimates on the density of states, Communications in Mathematical Physics. 338(2) (2015) 705-720.

36. J. D. Simoi, Fermi acceleration in anti-integrable limits, Communications in Mathematical Physics. 321 (2013) 703-745

37. J. D. Meiss, Visual explorations of dynamics: the Standard map, Pramana. 70(6) (2008) 965-988.

38. A. J. Lichtenberg and M. A. Lieberman, Regular and stochastic motion. (Springer, Berlin, Germany, 1983).

39. H.O.Peitgen, H. Jürgens and D. Saupe, Chaos and Fractals: New Frontiers of Science, Springer, 2004.

40. C. C. Chen, E. E. Wolf, E. Eduardo, et al. Deciphering and Controlling Intermittent Chaos on a Catalytic Wafer. American Control Conference, (San Francisco, CA, USA, 1993), 2922-2925. 